\title{
Stenosis and Dissection in the Same Vertebral Artery
}

\author{
Evguenia Vassileva ${ }^{\mathrm{a}, \mathrm{c}}$, Ivo Petrov ${ }^{\mathrm{b}}$, Evgenii Vavrek $^{\mathrm{a}}$
}

\begin{abstract}
In the current report, we present a case of a 59-year-old patient with arterial hypertension, diabetes and dislipidemia, which is presented by severe bilateral atherosclerotic lesions of the extracranial part of the vertebral arteries, thrombosis of the right and high-grade stenosis of the V1 segment of the left vertebral artery (VA). Despite the presence of the prominent atherosclerotic VA pathology, the patient develops asymptomatic VA dissection of the left V1 segment. The brain computed tomography was without data for brain infarction. The patient was without neurological deficit. Treatment with stent placement was performed.
\end{abstract}

Keywords: Vertebral artery; Vertebral stenosis; Vertebral dissection; Stenting

\section{Introduction}

Spontaneous cervical artery dissections are increasingly recognized as a common cause of ischemic stroke in young adults $[1,2]$, but they may occur in any age. The treatment of cervical artery dissection remains highly controversal. The diversity of treatment options reflects the huge heterogenity of spontaneous cervical artery dissections, linked to the association of several factors [3]. We present a case of a spontaneous vertebral artery (VA) dissection in a middle-aged patient treated by stent placement.

\footnotetext{
Manuscript accepted for publication April 11, 2014

aDepartment of Neurology, University Hospital "Tsaritsa

Yoanna - ISUL", Sofia, Bulgaria

bepartment of Cardiology, "City Clinic", Sofia, Bulgaria

'Corresponding author: Evguenia Vassileva, University Hospital

"Tsaritsa Yoanna - ISUL", Clinic of Neurology, 8 "Bialo More" Street,

1527 Sofia, Bulgaria. Email: iruschev@yahoo.com
}

doi: http://dx.doi.org/10.14740/jmc1774w

\section{Case Report}

A 59-year-old female patient with a history of one episode of syncope (10 days before the first visit to our department) was referred to color coded duplex sonography (CCDS) for a diagnostic work-up. Her medical history included arterial hypertension, diabetes and dislipidemia. Two years before she had coronary and iliac artery stenting and balloon angioplasty of the right subclavian artery. The patient was without neurological deficit. Brain computed tomography was without data for brain infarction.

CCDS revealed non-stenotic atherosclerotic plaques of both internal carotid arteries and thrombosis of the right VA. On her left VA two stenotic lesions were detected. The first was high-grade atherosclerotic stenosis in the origin of VA (Fig. 1).

The second lesion was at the distal part of V1 segment (Fig. 2).

The ultrasound findings of the second vertebral lesion were highly characteristic for VA dissection, hypodense intramural hematoma, causing eccentric stenosis and segmental arterial dilatation. The patient was referred for digital subtraction angiography and two stenotic lesions were revealed: left high-grade VA atherosclerotic stenosis and left VA dissection (Fig. 3).

The patient underwent endovascular stenting of extracranial VA. Follow-up CCDS 3 years after the intervention revealed normal blood flow of the left VA.

\section{Discussion}

The history of our patient revealed atherosclerosis at various sites in the vascular tree (coronary, subclavian, iliac arteries). Severe atherosclerosis of both VA (thrombosis of the right and high-grade stenosis of the left VA) as well as non-stenotic plaques of the carotid arteries was detected by CCDS.

Cervical artery dissection is considered as non-atherosclerotic vasculopathies causing cerebral ischemia, affecting mainly young individuals. Although the etiology of the spontaneous cervical artery dissection remains incompletely 


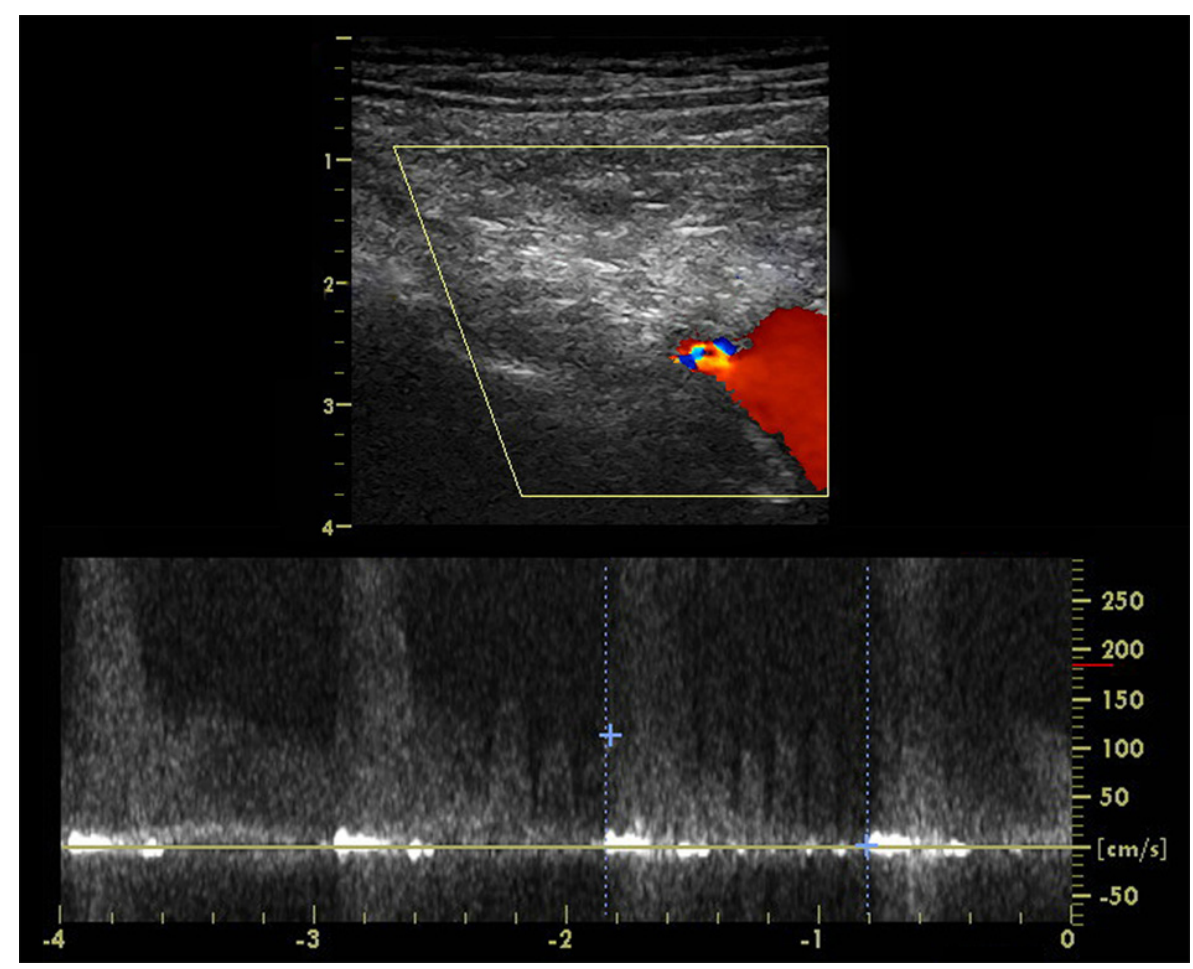

Figure 1. CCDS of the left VA, high-grade atherosclerotic stenosis in the origin of VA.

understood, an underlying structural defect of the extracellular matrix, resulting from a combination of genetic and environmental factors, is generally suspected [4]. Atherosclerosis appears to be rare in patients affected by cervical artery dissection [5]. Several studies investigated the potential link of cervical artery dissection with common risk factors for atherosclerosis [5-7]. In the largest study Debette et al [6] reported that hypertension was associated with an increased risk of cervical artery dissection, whereas an inverse associa- tion with hypercholesterolemia, obesity and overweight was observed. Vascular risk factor profile of patients suggests that atherosclerosis is probably not a predisposing condition to cervical artery dissection [6]. But the relationship between the atherosclerosis and the dissections of the cervical arteries is not examined up to now.

The clinical presentation of the spontaneous VA dissection is highly variable. Patients with spontaneous extracranial VA dissection may present with ischemic stroke, transient

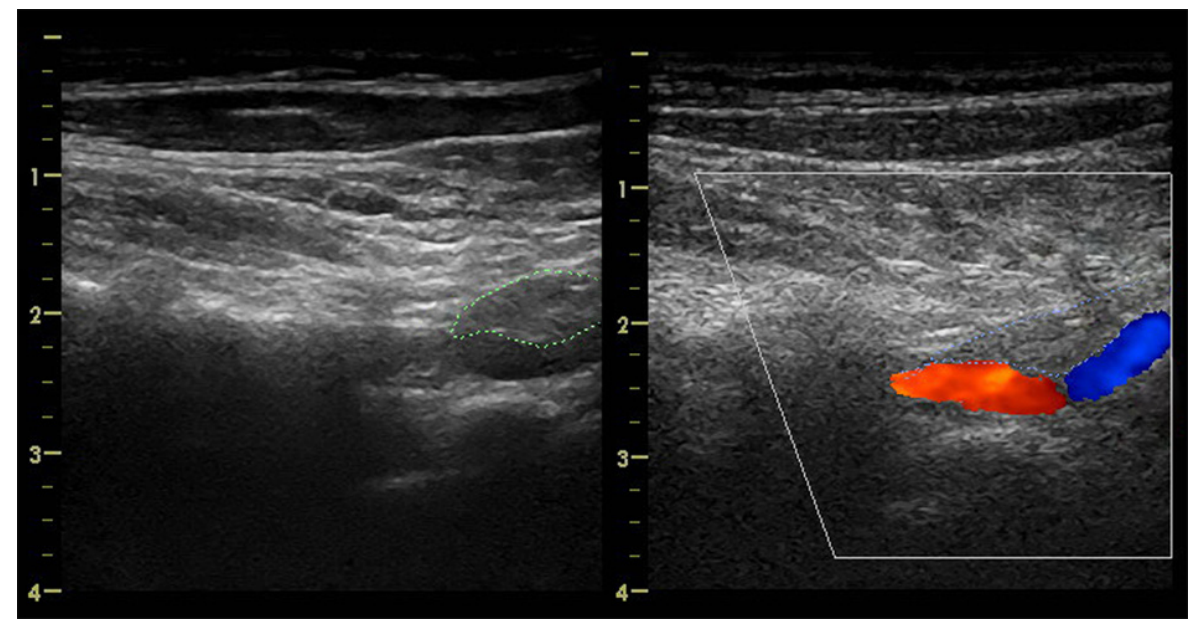

Figure 2. CCDS of the left VA, hypodense intramural hematoma, causing eccentric stenosis and segmental arterial dilatation consistent with VA dissection in V1 segment. 


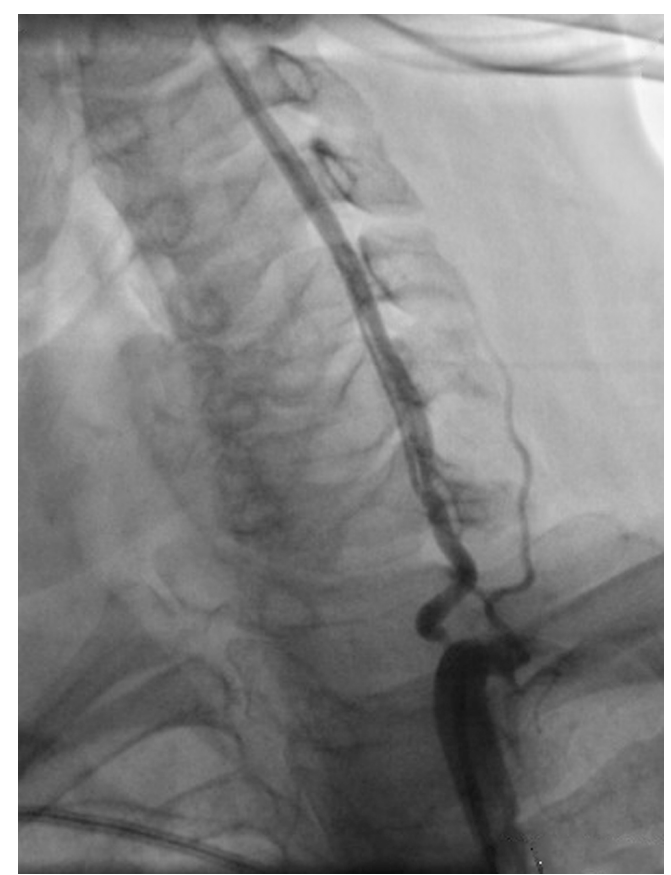

Figure 3. Digital subtraction angiography, high-grade VA atherosclerotic stenosis and VA dissection in V1 segment.

ischemic attack, sensorimotor cervical radiculopathy $\mathrm{C} 5 / \mathrm{C} 6$, pulsatile tinnitus or occipital head and neck pain alone [810]. Arnold et al reported asymptomatic VA dissections in $8 \%$ of 169 patients with VA dissection [8]. It is interesting that in our case asymptomatic VA dissection developed in patient with asymptomatic atherosclerotic bilateral high-grade VA obstructions. The absolute risk of posterior circulation stroke in patients with atherosclerotic arterial disease and asymptomatic VA origin stenosis is low [11]. The absence of neurologic deficits in our case could be attributed to the developed collateral pathways during the slowly progressive atherosclerotic process and the adequate intracranial collateral flow.

We emphasize on the highly diagnostic ultrasound possibilities to distinguish between the two types of VA stenotic lesions. Vertebral origin and near the origin is the most affected by atherosclerotic stenosis VA segment [1], while the entrance of the artery into the transverse foramen of the C6 vertebra is the most typical dissection site in V1 segment [12]. The first lesion is concentric and high-graded, while the second lesion is intramural hypoechogenic hematoma, causing eccentric moderate grade stenosis and local dilatation of arterial diameter.

In the recent years, treatment of VA stenosis by percutaneous transluminal angioplasty has been introduced [13, 14]. There were much less cases of application of this treatment in patients with VA dissection. The safety and benefit of stenting of the VA dissection as compared with the medical therapy are unclear. For VA dissection, the review of Pham et al [15] concerning all cases with VA dissection treated by stenting reported a $100 \%$ technical success rate and no neurological complication during the follow-up. The CCDS follow-up period of our patient was 3 years. No new neurological events during the follow-up period were observed.

\section{Conclusion}

VA dissection could be found in patient with cervical artery atherosclerosis. VA stenting could be presumed in such cases.

\section{References}

1. Leys D, Bandu L, Henon H, Lucas C, Mounier-Vehier F, Rondepierre P, Godefroy O. Clinical outcome in 287 consecutive young adults (15 to 45 years) with ischemic stroke. Neurology. 2002;59(1):26-33.

2. Arnold M, Halpern M, Meier N, Fischer U, Haefeli T, Kappeler L, Brekenfeld C, et al. Age-dependent differences in demographics, risk factors, co-morbidity, etiology, management, and clinical outcome of acute ischemic stroke. J Neurol. 2008;255(10):1503-1507.

3. Arnold M, Fischer U, Bousser MG. Treatment issues in spontaneous cervicocephalic artery dissections. Int J Stroke. 2011;6(3):213-218.

4. Brandt T, Hausser I, Orberk E, Grau A, Hartschuh W, Anton-Lamprecht I, Hacke W. Ultrastructural connective tissue abnormalities in patients with spontaneous cervicocerebral artery dissections. Ann Neurol. 1998;44(2):281-285.

5. Pezzini A, Caso V, Zanferrari C, Del Zotto E, Paciaroni M, Bertolino C, Grassi M, et al. Arterial hypertension as risk factor for spontaneous cervical artery dissection. A case-control study. J Neurol Neurosurg Psychiatry. 2006;77(1):95-97.

6. Debette S, Metso T, Pezzini A, Abboud S, Metso A, Leys $\mathrm{D}$, Bersano A, et al. Association of vascular risk factors with cervical artery dissection and ischemic stroke in young adults. Circulation. 2011;123(14):1537-1544.

7. Arnold M, Pannier B, Chabriat H, Nedeltchev K, Stapf C, Buffon F, Crassard I, et al. Vascular risk factors and morphometric data in cervical artery dissection: a case-control study. J Neurol Neurosurg Psychiatry. 2009;80(2):232-234.

8. Arnold M, Bousser MG, Fahrni G, Fischer U, Georgiadis D, Gandjour J, Benninger D, et al. Vertebral artery dissection: presenting findings and predictors of outcome. Stroke. 2006;37(10):2499-2503.

9. Schievink WI. Spontaneous dissection of the carotid and vertebral arteries. N Engl J Med. 2001;344(12):898-906.

10. Arnold M, Bousser MG. Clinical manifestations of vertebral artery dissection. Front Neurol Neurosci. 
2005;20:77-86.

11. Compter A, van der Worp HB, Algra A, Kappelle LJ, Second Manifestations of AdSG. Prevalence and prognosis of asymptomatic vertebral artery origin stenosis in patients with clinically manifest arterial disease. Stroke. 2011;42(10):2795-2800.

12. Bartels E, Flugel KA. Evaluation of extracranial vertebral artery dissection with duplex color-flow imaging. Stroke. 1996;27(2):290-295.

13. Eberhardt O, Naegele T, Raygrotzki S, Weller M, Ernemann U. Stenting of vertebrobasilar arteries in symptomatic atherosclerotic disease and acute occlusion: case series and review of the literature. J Vasc Surg.
2006;43(6):1145-1154.

14. Coward LJ, McCabe DJ, Ederle J, Featherstone RL, Clifton A, Brown MM, Investigators C. Long-term outcome after angioplasty and stenting for symptomatic vertebral artery stenosis compared with medical treatment in the Carotid And Vertebral Artery Transluminal Angioplasty Study (CAVATAS): a randomized trial. Stroke. 2007;38(5):1526-1530.

15. Pham MH, Rahme RJ, Arnaout O, Hurley MC, Bernstein RA, Batjer HH, Bendok BR. Endovascular stenting of extracranial carotid and vertebral artery dissections: a systematic review of the literature. Neurosurgery. 2011;68(4):856-866; discussion 866 . 